\title{
Benefícios psicológicos do slackline: relação entre estado de fluxo, tempo de prática e qualidade de vida
}

\section{RESUMO}

Esse estudo teve o objetivo de avaliar o nível de Estado de Fluxo de praticantes de slackline e relacioná-lo com a qualidade de vida dos indivíduos. Foram avaliados 30 sujeitos com idade média de $22( \pm 3)$ anos. Os instrumentos utilizados: Ficha de identificação; Flow State Scale-2 (FSS-2), Dispositional Flow Scale-2 (DFS-20) e World Health Organization Quality of Life-Bref (WHOQoL-BREF). Para verificar a correlação entre os instrumentos utilizou-se o coeficiente de Spearman, no qual foi possível observar elevados níveis de Estado de Fluxo e qualidade de vida geral. Foi encontrada correlação significativa entre $\mathrm{o}$ fator psicológico da qualidade de vida com a disposição para atingir o Fluxo juntamente com o tempo de prática e duração da sessão de treino. Concluímos que a prática do slackline possibilita o desenvolvimento de aspectos psicológicos, como o Estado de Fluxo e com isso possibilita a melhora na qualidade de vida de seus praticantes.

PALAVRAS-CHAVE: Slackline; Estado de fluxo; Psicologia do esporte; Qualidade de vida
Juliano Marques Franco

Licenciatura e Bacharel em Educação Física

Universidade Estadual de Campinas UNICAMP, FEF, Campinas, São Paulo, Brasil Julianofranco4@gmail.com (1) https://orcid.org/0000-0002-1825-0097

Rosane Camila de Godoi Bacharel em Educação Física Universidade Estadual de Campinas UNICAMP, FEF, Campinas, São Paulo, Brasil r.camilagodoi@gmail.com

https://orcid.org/0000-0003-1229-992X

Hélio Mamoru Yoshida

Mestre em Educação Física Universidade Estadual de Campinas UNICAMP, FEF, Campinas, São Paulo, Brasil heliomyoshida@gmail.com https://orcid.org/0000-0002-1189-9192

Fernanda Tartalha do Nascimento

Mestranda em Educação Física Universidade Estadual de Campinas UNICAMP, FEF, Campinas, São Paulo, Brasil fertartalha@gmail.com

https://orcid.org/0000-0001-5166-1229

Paula Teixeira Fernandes Professora Livre-docente do Departamento de Ciências do Esporte Universidade Estadual de Campinas UNICAMP, FEF, Campinas, São Paulo, Brasil paula@fefunicamp.br https://orcid.org/0000-0002-0492-1670 


\title{
Psychological benefits of slackline: relationship between flow state, time of practice and quality of life
}

\begin{abstract}
This study aimed to assess the level of Flow State of slackline practitioners and to relate it to the their quality of life. We evaluated 30 subjects with mean age of $22( \pm 3)$ years. The instruments used: Identification sheet; Flow State Scale-2 (FSS-2), Dispositional Flow Scale-2 (DFS-20) and World Health Organization Quality of Life-Bref (WHOQoL-BREF). To verify the correlation between the instruments the Spearman coefficient was used, in which it was possible to observe high levels of Flow State and general quality of life. A significant correlation was found between the psychological quality of life factor and the disposition to reach the Flow along with the practice time and duration of the training session. We conclude that the practice of slackline allows the development of psychological aspects, such as the Flow State and with that allows the improvement in the quality of life of its practitioners.
\end{abstract}

KEYWORDS: Slackline; Flow state; Sports psychology; Quality of life

\section{Beneficios psicológicos del slackline: relación entre estado de flujo, tiempo de práctica y calidad de vida}

\section{RESUMEN}

Este estudio tuvo el objetivo de evaluar el nivel de Estado de Flujo de practicantes de slackline y relacionarlo con la calidad de vida de los individuos. Se evaluaron 30 sujetos con edad media de 22 ( \pm 3) años. Los instrumentos utilizados: Ficha de identificación; Flow State Scale-2 (FSS-2), Dispositional Flow Scale-2 (DFS-20) e World Health Organization Quality of Life-Bref (WHOQoL-BREF). Para verificar la correlación entre los instrumentos se utilizó el coeficiente de Spearman, en el cual fue posible observar elevados niveles de Estado de Flujo y calidad de vida general. Se encontró correlación significativa entre el factor psicológico de la calidad de vida con la disposición para alcanzar el Flujo junto con el tiempo de práctica. Concluimos que la práctica del slackline posibilita el desarrollo de aspectos psicológicos, como el Estado de Flujo y con ello posibilita la mejora en la calidad de vida de sus practicantes.

PALABRAS-CLAVE: Slackline; Estado de flujo; Psicología del deporte; Calidad de vida 


\section{INTRODUÇÃO}

Esporte e Psicologia começaram a ter relação mais estreita no final do século XIX e início do século XX. Este diálogo culminou em uma área conhecida hoje como Psicologia do Esporte (SOUZA; SOUZA; FERREIRA, 2011). Segundo Weinberg e Gould (2017), os estudos nessa área têm dois objetivos: (1) entender como fatores psicológicos afetam o rendimento dos indivíduos e (2) entender como a participação em práticas esportivas influencia o desenvolvimento psicológico, a saúde e o bem-estar dos indivíduos.

Neste sentido, são estudados diversos fatores psicológicos, tanto positivos quanto negativos, buscando entender a interação destes com a prática esportiva. Jackson (1996) aponta que, tanto as experiências positivas, quanto as negativas são igualmente importantes de serem estudadas e entendidas, pois dependem de cada pessoa, de cada situação e de cada modalidade esportiva.

Dentre as variáveis psicológicas, o Estado de Fluxo é considerado como o estado positivo em que o indivíduo está totalmente conectado à atividade, sendo, portanto, um estado desejado por atletas, que pode ser desfrutado por qualquer praticante de atividade física (JACKSON, 1996). Segundo Zumeta e colaboradores (2016), o Estado de Fluxo é uma experiência ótima e profundamente gratificante, com foco intenso na atividade, sendo que a pessoa é totalmente absorvida por esta, eliminando todos os outros pensamentos e emoções.

Csikszentmihalyi (1999) desenvolveu a teoria sobre o Estado de Fluxo, a partir da investigação de atividades intrinsicamente motivadoras na década de 60 . O autor estudou indivíduos que realizavam escalada, dança, xadrez, música e esportes, sempre visando identificar as características necessárias para atingir este estado 'ótimo' (NAKAMURA \& CSIKSZENTMIHALYI, 2009).

Csikszentmihalyi e colaboradores, no estudo publicado em 2018, abordam o Estado de Fluxo como combustível motivacional em atividades criativas. Este estado psicologicamente ótimo é capaz de motivar o indivíduo na busca de metas criativas e também de dar soluções mais criativas para desafios percebidos (CSIKSZENTMIHALYI, MONTIJO, MOUNTON, 2018).

O Estado de Fluxo correlaciona-se positivamente com o rendimento esportivo (JACKSON \& ROBERTS, 1992) e também com maior prazer e motivação para a prática (JACKSON, 1996). No esporte, atingir o Estado de Fluxo é de extrema importância, uma vez que representa o sentimento de profundo bem-estar, sem interferência de outras manifestações psicológicas negativas pertencentes, muitas vezes, à vida cotidiana ou ao estresse da própria prática a que está inserido.

Para acessar o Estado de Fluxo é necessário alguns elementos:

1. Percepção do desafio proposto; 
2. Objetivo definido;

3. Consciência da qualidade das suas ações, ou seja, um feedback imediato;

4. Concentração e envolvimento profundo com a tarefa;

5. Sensação de controle sobre situações difíceis;

6. Foco apenas no momento presente;

7. Alteração na percepção da passagem do tempo, uma impressão de que o tempo passou mais devagar, ou mais rapidamente que o de costume;

8. Equilíbrio entre suas habilidades e o desafio proposto;

9. Fusão entre ação e consciência, de modo que não há energia extra sendo despendida em outra tarefa naquele momento.

Essas características foram moduladas pelos principais autores do tema, Csikszentmihalyi, Jackson e Nakamura, nos estudos que se estenderam de 1996 a 2002 nas quais algumas dessas características são fundamentais para caracterizar o Fluxo. Por exemplo, ser capaz de perceber o feedback e se adaptar de acordo com o desempenho na tarefa pode proporcionar a atenção necessária para um Estado de Fluxo mais profundo num processo cíclico (LAMBERT \& CSIKSZENTMIHALYI, 2019)

Segundo esses autores há, ainda, um outro elemento que possibilita a ocorrência de todos os demais: a experiência autotélica (auto: por si mesmo/de si mesmo; e télico: finalidade). Neste sentido, a experiência deve ter um fim em si mesma, não tendo outra recompensa que o simples prazer de realizá-la (CSIKSZENTMIHALYI, 1990).

A Teoria do Fluxo mostra o ser-humano em uma perspectiva de constante aprendizado, resultando em um desenvolvimento contínuo de habilidades à medida que o desafio se torna mais fácil e suas habilidades mais apuradas. Essa relação estabelece mudanças para que o Fluxo se mantenha presente, provocando um processo de desenvolvimento humano que se dá por toda a vida (TSE, NAKAMURA, CSIKSZENTMIHALYI, 2019).

Assim, conhecer o Estado de Fluxo e a disposição que os praticantes têm para acessá-lo pode colaborar com o bom rendimento em diversas práticas, circunscritas tanto no meio esportivo quanto em outros âmbitos da vida. Buscar atividades de cunho autotélico passou a ser mais comum e o slackline é um exemplo disso.

O slackline é uma modalidade esportiva, na qual o objetivo é manter-se em equilíbrio em pé ou andando em cima de uma fita (KELLER et al., 2012). Dependendo do tamanho e da tensão da fita, as características da prática são alteradas e o nível de dificuldade ajustado (PFUSTERSCHMIED et al., 2013). Esta modalidade é considerada um esporte de aventura (PEREIRA, 2013) e vem ganhando mais adeptos nos últimos anos (XAVIER, 2012). 
A literatura científica sobre a modalidade é recente, sendo que a maioria dos estudos foca nos efeitos posturais e no equilíbrio promovido pela prática (DONATH et al., 2013; GRANACHER et al., 2010; KELLER et al., 2012; PFUSTERSCHMIED et al., 2013; THOMAS \& KALICINSKI, 2016). Até o presente momento, nenhum estudo investigou a relação do Estado de Fluxo em praticantes de slackline, tampouco relacionando à qualidade de vida (QV), que é definida pela Organização Mundial da Saúde (OMS, 1946) como a autopercepção do indivíduo sobre sua saúde, o contexto sociocultural em que está inserido levando em consideração seus objetivos, expectativas e padrões pessoais. Sendo assim, a qualidade de vida reflete a subjetividade do resultado e sua compreensão sobre sua própria vida (ROPKE et al, 2017) podendo ser avaliada utilizando o WHOQOL-bref (The WHOQOL Group, 1998), que mensura a qualidade de vida em quatro Domínios: o Físico; o Social; o Psicológico e o de Meio Ambiente.

Diante deste contexto, o objetivo deste estudo foi constatar a presença do Estado de Fluxo nesse público, a disposição que estes indivíduos têm em acessá-lo para, posteriormente, correlacionar os achados com o tempo de prática, a frequência na semana, a duração da sessão de treino e a qualidade de vida.

\section{MÉTODOS}

Esta pesquisa foi aprovada pelo Comitê de Ética em Pesquisa da UNICAMP (CAAE: 24439313.7.0000.5404), respeitando os princípios da declaração de Helsinque (WORLD MEDICAL ASSOCIATION, 2001). Todos os participantes deixaram claro sua concordância com a pesquisa através da assinatura do Termo de Concentimento Livre e Esclarecido (TCLE).

A pesquisa foi realizada em locais de prática de slackline das cidades de Campinas e Bragança Paulista, no interior do estado de São Paulo, Brasil.

\section{Amostra}

Participaram da pesquisa 30 praticantes de slackline, 24 homens e 6 mulheres, com média de idade de $22 \pm 3$ anos. Como critério de inclusão, os sujeitos deveriam ser maiores de 18 anos e ter no mínimo 3 meses de prática, com frequência de pelo menos 1 vez por semana. Os sujeitos que possuíram algum déficit associado que impedisse a resposta aos questionários e/ou que não aceitassem participar da pesquisa por meio da assinatura do Termo de Consentimento Livre e Esclarecido (TCLE) foram excluídos do estudo. 


\section{Instrumentos}

Foram utilizados quatro questionários:

1) Ficha de identificação para caracterização geral do praticante, com informações quanto à idade, sexo, participação em competição, tempo de prática, frequência de prática por semana e duração da sessão em horas.

2) Flow State Scale-2 (FSS-2) (JACKSON \& EKLUND, 2002) que avalia o acesso à experiência de Fluxo em atividades físicas. É respondido logo após a prática justamente por mensurar o Estado de Fluxo especificamente em uma atividade. É composto por 9 questões, onde cada uma remete a uma dimensão do Estado de Fluxo.

3) Dispositional Flow Scale-2 (DFS-2) (JACKSON \& EKLUND, 2002) que avalia a disposição/propensão de o sujeito acessar o Estado de Fluxo a partir da frequência com que o sujeito experimenta este estado em sua prática. Deve ser respondido antes da prática. Este instrumento é composto por 9 questões, onde cada uma remete a uma dimensão Estado de Fluxo.

Cada questão do DFS-2 e do FSS-2 possui uma escala de 1 a 5. Para o cálculo final, somamse os resultados de cada questão e divide-se por 9, isto é, uma média simples. Sendo assim, quanto mais perto de 5 a média, maior é o índice de Fluxo.

4) World Health Organization Quality of Life - Bref (WHOQOL-Bref) (WORLD HEALTH ORGANIZATION, 1996) que contém questões para a qualidade de vida nos domínios: físico, psicológico, relações sociais e meio ambiente. Contém 26 questões, sendo duas questões gerais de qualidade de vida, e as demais representam cada uma das 24 facetas que compõem o instrumento original. Para esse estudo, foi considerada a pontuação entre 0 e 100 (PEDROSO et al., 2010).

\section{Procedimentos}

Foi apresentado o projeto e seus objetivos para os voluntários que aceitaram participar da pesquisa. Após assinarem o TCLE, responderam aos questionários individualmente. As condições de aplicação foram semelhantes para todos. Os participantes levaram aproximadamente 20 minutos para responder os instrumentos: Ficha de identificação, DFS-2 e WHOQOL-Bref, aplicados antes da prática do slackline, e 3 minutos para responder ao último, FFS-2, após a prática. 


\section{Análise estatística}

Foi realizada a análise exploratória de dados através de medidas resumo (frequência, porcentagem, média, desvio padrão ( $\pm \mathrm{DP})$, mínimo, mediana e máximo). A correlação entre os instrumentos foi avaliada através do coeficiente de Spearman. O teste não paramétrico de KruskalWallis foi usado para comparar os grupos de diferentes níveis em relação aos instrumentos.

\section{RESULTADOS}

O estudo teve o objetivo de avaliar o Estado de Fluxo nos praticantes de slackline e relacionar estes dados com a $\mathrm{QV}$, tempo de prática, frequência na semana e duração da sessão de treino.

Participaram do estudo 30 sujeitos. Os praticantes tiveram tempo médio de 1 ano $( \pm 1,06)$, com frequência de $2( \pm 1,31)$ vezes na semana, com duração de $3( \pm 1,33)$ horas.

Estão presentes na Tabela 1, os valores dos instrumentos para o acesso ao Estado de Fluxo (FSS-2), a disposição/propensão para atingir Estado de Fluxo (DFS-2) e os índices do WHOQOLbref.

Tabela 1: Valores Estado de Fluxo e Índices de Qualidade de Vida.

\begin{tabular}{lccccc}
\hline Questionário (n=30) & Média & DP $( \pm)$ & Mínimo & Mediana & Máximo \\
\hline FSS-2 & 3,97 & 0,48 & 3,00 & 4,00 & 5,00 \\
DFS-2 & 3,92 & 0,49 & 2,80 & 4,00 & 4,70 \\
\hline Domínios da Qualidade de Vida (n=21) & & & & & \\
\hline Físico & 71,77 & 10,94 & 50,00 & 71,43 & 89,29 \\
Psicológico & 73,02 & 12,95 & 41,67 & 79,17 & 91,67 \\
Social & 75,79 & 12,61 & 50,00 & 75,00 & 100,00 \\
Ambiente & 61,61 & 10,88 & 43,75 & 62,5 & 81,25 \\
Qualidade de Vida Geral & 70,55 & 07,75 & 57,55 & 71,91 & 86,68 \\
\hline
\end{tabular}

Legenda: $\mathrm{n}=$ Número total de respostas; DP=Desvio Padrão. FSS-2: Flow State Scale (escala de 1 a 5 ); DFS-2: Dispositional Flow Scale (escala de 1 a 5). Escala WHOQOL breve para escore entre 0 e 100.

A Tabela 2 apresenta a correlação entre os índices da qualidade de vida, Estado de Fluxo, disposição para acessar o Estado de Fluxo, tempo de prática do slackline, duração da sessão e quantidade por semana. O Estado de Fluxo correlacionou-se moderadamente com a duração da sessão $(r=0,46)$. 
No que concerne à disposição para se atingir o Estado de Fluxo (DFS-2) verificou-se uma correlação positiva moderada com a duração da sessão $(\mathrm{r}=0,50)$ e com o domínio psicológico da $\mathrm{QV}(\mathrm{r}=0,51)$.

O domínio psicológico da QV também se correlacionou moderadamente com o tempo de prática $(\mathrm{r}=0,53)$ e a duração da sessão $(\mathrm{r}=0,58)$. O domínio meio ambiente da $\mathrm{QV}$ se correlacionou moderadamente com a duração da sessão $(\mathrm{r}=0,48)$.

Tabela 2: Correlação entre Estado de Fluxo, Qualidade de Vida e Tempo de Prática de Slackline.

\begin{tabular}{lccccc}
\hline & FSS-2 & DFS-2 & $\begin{array}{c}\text { TEMPO DE } \\
\text { PRÁTICA }\end{array}$ & X/SEMANA & DURAÇÃO (h) \\
\hline WHOQOL FIS & 0,37 & 0,36 & $-0,018$ & 0,11 & 0,16 \\
WHOQOL PSI & 0,36 & $\mathbf{0 , 5 1 *}$ & $\mathbf{0 , 5 3 *}$ & 0,41 & $\mathbf{0 , 5 8 *}$ \\
WHOQOL SOC & 0,33 & 0,40 & 0,16 & 0,22 & 0,14 \\
WHOQOL M.A. & 0,23 & 0,34 & 0,27 & 0,02 & $\mathbf{0 , 4 8 *}$ \\
FSS-2 & - & $\mathbf{0 , 7 8 *}$ & 0,26 & $-0,03$ & $\mathbf{0 , 4 6 *}$ \\
DFS-2 & $\mathbf{0 , 7 8 *}$ & - & 0,27 & 0,11 & $\mathbf{0 , 5 0 *}$ \\
\hline
\end{tabular}

Legenda: DFS-2=Dispositional Flow Scale; FSS-2=Flow State Scale; WHOQOL FIS=Domínio Físico; WHOQOL PSI=Domínio Psicológico; WHOQOL SOC=Domínio Social; WHOQOL M.A=Domínio Meio Ambiente; Tempo Prática=Há quanto tempo pratica; X/Semana=Vezes na Semana; Duração $(\mathrm{h})=$ Duração da sessão em horas.* Correlações Estatisticamente Significativas, $\mathrm{p}<0,05$.

\section{DISCUSSÃO}

$\mathrm{Na}$ literatura estudada, é possível notar ausência de pesquisas que investigam aspectos psicológicos e de qualidade de vida em praticantes de slackline, o que torna esse estudo inovador. Foram encontradas correlações entre as variáveis estudadas, que permitem um maior conhecimento sobre a modalidade, porém a escassez de estudos na área tornou limitado o diálogo com a literatura existente.

Durante a atividade, o Estado de Fluxo relaciona-se ao bom desempenho, vivência positiva e maior prazer e motivação para a atividade (JACKSON, EKLUND, 2002; JACKSON, ROBERTS, 1992; WEINBERG, GOULD, 2017), podendo beneficiar tanto atletas de alto rendimento, quanto praticantes recreativos.

No que diz respeito a qualidade de vida, que de acordo com a Constituição Mundial da Saúde (1946) se faz de extrema importância e constitui um dos direitos fundamentais de todo ser humano, encontrou-se uma correlação positiva entre as respostas obtidas no DFS-2 e as respostas do WHOQOL no Domínio Psicológico, estes achados corroboram com Vieira et al (2011), em que os esportes de aventura produzem experiências gratificantes, sentimentos de prazer e de satisfação pessoal, pois há o equilíbrio entre a capacidade pessoal e dificuldade de execução dos movimentos, 
tornando uma tarefa intrinsecamente motivadora. Nesse mesmo sentido, Csikszentmihalyi (1999b) afirma que a motivação intrínseca está potencialmente relacionada com o Estado de Fluxo. Um cenário desse permite um diálogo com o que Sodré e colaboradores (2017) afirmam quando dizem que a prática do slackline auxilia na melhora da concentração e alívio do estresse.

A correlação entre o Domínio Psicológico, tempo de prática e a duração da sessão de treino corroboram com o estudo de Oliveira e Miranda (2015) e pode ser explicado por Neto e Kendrick (2014), pois o desafio de executar ações motoras em cima de uma linha exige o foco e concentração, motivando os atletas a persistirem na prática até atingir o sucesso, o que promove uma conversa entre o prazer da prática (Domínio Psicológico), buscar o êxito e, assim, ter um maior tempo de prática, com consequente aumento da duração da sessão.

O Domínio Meio Ambiente do WHOQOL também mostrou correlação positiva com a duração da prática. Os esportes de aventura propiciam a interação com o meio em que se pratica. $\mathrm{O}$ slackline, enquanto parte deste âmbito de atividades, promove esta interação, uma vez que muitos sujeitos procuram praças arborizadas, montanhas, lagos, rios entre outros locais (BARROS, 2015), portanto, quanto maior a duração da prática do slackline, maior contato com o meio em que se está inserido.

A prática de atividade física, por sua vez, pode influenciar no aumento da qualidade de vida das pessoas se comparado às sedentárias, o que indica uma associação positiva entre atividade física e qualidade de vida (FERREIRA, 2015), mostrando que os benefícios do slackline vão além do acesso ao Estado de Fluxo.

Além desta observação, destacamos a importância da duração da sessão de slackline, pois este fator correlacionou-se positivamente com o FSS-2, DFS-2 e o Domínio Psicológico da QV. As análises de correlação não permitem definir o processo de causa e efeito das variáveis analisadas, contudo, esse estudo sugere como hipótese para futuras investigações que o sujeito com maior disposição em acessar o Estado de Fluxo tem maior propensão em manter a duração da sessão, promover a relação com seu meio ambiente, e assim acessar o Estado de Fluxo, melhorando a qualidade de vida.

O Estado de Fluxo está associado a situações que envolvem novidade, variação de resultados e atividades de exploração, enquanto a experiência é descrita como agradável envolvendo até mesmo menor percepção de esforço e efeito energizante (SWANN, 2019), dessa forma, fica evidente associar a prática de slackline ao Estado de Fluxo. 


\section{CONCLUSÕES}

O presente estudo, por ser pioneiro na área da Psicologia do Esporte, possibilitou a análise dos praticantes de slackline, trazendo luz para a compreensão dos níveis de Estado de Fluxo e suas correlações com a qualidade de vida.

Esta pesquisa revelou que praticantes de slackline possuem níveis elevados de Estado de Fluxo, assim como alta disposição para atingi-lo e níveis também elevados de qualidade de vida. Ressaltamos que a duração da sessão de treino/prática correlacionou-se com componente psicológico e meio ambiente da qualidade de vida, sugerindo que quanto mais tempo o sujeito passa praticando o slackline, maior é a sua satisfação psicológica e com o meio ambiente ao seu redor.

E por fim, nossa expectativa é possibilitar, à comunidade científica e aos interessados no tema, a reflexão dos conhecimentos acadêmicos sobre o slackline, ainda pouco explorado, enfatizando a importância do componente psicológico do Estado de Fluxo e os benefícios para a qualidade de vida nesta modalidade.

\section{REFERÊNCIAS}

BARROS, Daniel. O slackline: do surgimento a evolução e seus benefícios. Revista digital EFDeportes, v. 19, n. 202, 2015.

CONSTITUIÇÃO DA ORGANIZAÇÃO MUNDIAL DA SAÚDE (OMS/WHO). Biblioteca Virtual de Direitos Humanos da USP. Nova Iorque, 1946. Disponível em: http://www.direitoshumanos.usp.br/index.php/OMS-Organiza\%C3\%A7\%C3\%A3o-Mundial-daSa\%C3\%BAde/constituicao-da-organizacao-mundial-da-saude-omswho.html. Acesso em: 20 de Abril de 2019.

CSIKSZENTMIHALYI, Mihaly. Flow: The psychology of optimal experience. Harper, 1990.

CSIKSZENTMIHALYI, Mihaly. A psicologia da felicidade. Saraiva. São Paulo, 1999a.

CSIKSZENTMIHALYI, Mihaly. A descoberta do fluxo: a psicologia do envolvimento com a vida cotidiana. Rocco. Rio de Janeiro, 1999b.

CSIKSZENTMIHALYI, Mihaly; MONTIJO, Monica; MOUNTON, Angela. Flow Theory: Optimizing Elite Performance in the Creative Realm. American Psychological Association. Chapter 14, pp 215 - 229. Washington, DC, United States, 2018.

DONATH, Lars. et al. Effects of slackline training on balance, jump performance \& muscle activity in young children. International journal of sports medicine, v. 34, n. 12, p. 1093-1098, 2013. 
FERREIRA, Joel Saraiva. et al. Influência da prática de atividade física sobre a qualidade de vida de usuários do SUS. Saúde Debate, vol. 39, nº 106, pp. 792-801. Rio de Janeiro, 2015.

GRANACHER, Urs. et al. Slackline training for balance and strength promotion. International journal of sports medicine, v. 31, n. 10, p. 717-723, 2010.

JACKSON, Susan. Toward a conceptual understanding of the flow experience in elite athletes. Research quarterly for exercise and sport. V. 67, n. 1, p. 76-90, 1996.

JACKSON, Susan; EKLUND, Robert. Assessing flow in physical activity: The flow state scale-2 and dispositional flow scale-2. Journal of Sport and Exercise Psychology. V. 24, n. 2, p. 133-150, 2002.

JACKSON, Susan; ROBERTS, Glyn. Positive performance states of athletes: Toward a conceptual understanding of peak performance. The Sport Psychologist. V. 6, n. 2, p. 156-171, 1992.

KELLER, Martin. et al. Improved postural control after slackline training is accompanied by reduced $\mathrm{H}$ reflexes. Scandinavian journal of medicine \& science in sports, v. 22, n. 4, p. 471-477, 2012.

LAMBERT, Joseph; CSIKSZENTMIHALYI, Mihaly. Facilitating or foiling flow: the role of momentary perceptions of feedback. The Journal of Positive Psychology. 2019.

MONTEIRO NETO, Jahyr Kendrick. Em busca do equilíbrio! slackline: da descoberta da prática à constituição de uma associação na cidade de Guaratuba. 2014.

NAKAMURA, Jeanne; CSIKSZENTMIHALYI, Mihaly. The Concept of Flow. Handbook of positive psychology, Chapter 7, 2002.

NAKAMURA, Jeanne; CSIKSZENTMIHALYI, Mihaly. Flow theory and research. Handbook of positive psychology, p. 195-206, 2009.

OLIVEIRA, Helder Zimmermann; MIRANDA, Renato. O estado de flow no basquetebol em categorias de base: uma análise da interferência da idade e do tempo de prática. Journal of Physical Education. V. 26, n. 1, p. 89-96, 2015.

PEDROSO, Bruno; PILATTI, Luiz Alberto; GUTIERREZ, Gustavo Luis; PICINI, Claudia Tania. Cálculo dos escores e estatística descritiva do WHOQOL-bref através do Microsoft Excel. Revista brasileira de qualidade de vida. V. 2, n. 1, 2010.

PEREIRA, Dimitri Wuo. SLACKLINE: Vivências acadêmicas na educação física. Motrivivência. V. 25, n. 41, p. 223-233, 2013.

PFUSTERSCHMIED, Jürgen. et al. Supervised slackline training improves postural stability. European Journal of Sport Science, v. 13, n. 1, p. 49-57, 2013.

ROPKE, Lucilene Maria. et al. Efeito da atividade física na qualidade do sono e qualidade de vida: revisão sistematizada. Arch Health Invest, vol. 6, pp. 561-566. 2017. 
SODRÉ, João Guilherme Hiroshi Higa. et al. Caracterização do perfil dos praticantes de Slackline no Brasil. Journal of Sport Pedagogy and Research, v. 3, n. 1, p. 4-12, 2017.

SOUZA, Simone.; SOUZA, Linton.; FERREIRA, Juliane. Psicologia do Esporte: surgimento, evolução e consolidação. Revista EFDeportes, n. 161, 2011.

SWANN, Christian; JACKMAN, Patricia; SCHWEICKL, Matthew; VELLA, Stewart. Optimal experiences in exercise: A qualitative investigation of flow and clutch states. Psychology of Sport \& Exercise. No 40, pp 87-98, 2019.

THOMAS, Monika.; KALICINSKI, Michael. The effects of slackline balance training on postural control in older adults. Journal of aging and physical activity, v. 24, n. 3, p. 393-398, 2016.

TSE, Dwight; NAKAMURA, Jeanne; CSIKSZENTMIHALYI, Mihaly. Beyond challenge-seeking and skill-building: Toward the lifespan developmental perspective on flow theory. The Journal of Positive Psychology. 2019.

VIEIRA, Lenamar Fiorese et al. Estado de fluxo em praticantes de escalada e skate downhill.

Motriz. Rio Claro, v. 17, n. 4, p. 591-599, 2011.

WEINBERG, Robert. \& GOULD, Daniel. Foundations of Sport and Exercise Psychology, Fifth Edition. [s.l: s.n.]. 2017

WHOQOL Group. The development of the World Health Organization quality of life assessment instrument (the WHOQOL). In: Orley, John., Kuyken, Willem. editors. Quality of life assessment: international perspectives. Heidelberg: Springer Verlag, 1994.

WORLD MEDICAL ASSOCIATION. World Medical Association Declaration of Helsinki. Ethical principles for medical research involving human subjects. Bulletin of the World Health Organization. V. 79, n. 4, p. 373, 2001.

WORLD HEALTH ORGANIZATION. WHOQOL-BREF: Introduction, administration, scoring and generic version of the assessment: field trial version, 1996.

XAVIER, Gabriele Carapeto. Slackline em Porto Alegre: configurações da prática. 2012.

ZUMETA, Larraitz; ORIOL, Xavier; TELLETXEA, Saioa; AMUTIO, Alberto; BASABE, Nekane. Collective efficacy in sports and physical activities: Perceived emotional synchrony and shared flow. Frontiers in psychology. V. 6, 2016.

\section{NOTAS DE AUTOR}

\section{AGRADECIMENTOS}

Os autores do estudo agradecem a Julia Barreira pelo auxílio nas análises de dados.

CONTRIBUIÇÃO DE AUTORIA

Concepção do manuscrito: J.M. Franco, R. C. Godoi, H.M. Yoshida, P. T. Fernandes

Coleta de dados: J.M. Franco.

Análise de dados: H.M. Yoshida 
Discussão dos resultados: J.M. Franco, H.M. Yoshida, P. T. Fernandes, F. T. Nascimento

Produção do texto: R. C. Godoi, H.M. Yoshida, P. T. Fernandes, F. T. Nascimento

Revisão e aprovação: H.M. Yoshida, P. T. Fernandes

\section{FINANCIAMENTO}

Não se aplica.

\section{CONSENTIMENTO DE USO DE IMAGEM}

Não se aplica.

\section{APROVAÇÃO DE COMITÊ DE ÉTICA EM PESQUISA}

Esta pesquisa foi aprovada pelo Comitê de Ética em Pesquisa da UNICAMP (CAAE: 24439313.7.0000.5404), respeitando os princípios da declaração de Helsinque.

\section{CONFLITO DE INTERESSES}

Não se aplica.

\section{LICENÇA DE USO}

Os autores cedem à Motrivivência - ISSN 2175-8042 os direitos exclusivos de primeira publicação, com o trabalho simultaneamente licenciado sob a Licença Creative Commons Attribution Non-Comercial ShareAlike (CC BY-NC SA) 4.0 International. Esta licença permite que terceiros remixem, adaptem e criem a partir do trabalho publicado, desde que para fins não comerciais, atribuindo o devido crédito de autoria e publicação inicial neste periódico desde que adotem a mesma licença, compartilhar igual. Os autores têm autorização para assumir contratos adicionais separadamente, para distribuição não exclusiva da versão do trabalho publicada neste periódico (ex.: publicar em repositório institucional, em site pessoal, publicar uma tradução, ou como capítulo de livro), com reconhecimento de autoria e publicação inicial neste periódico, desde que para fins não comerciais e compartilhar com a mesma licença.

\section{PUBLISHER}

Universidade Federal de Santa Catarina. Programa de Pós-Graduação em Educação Física. LaboMídia - Laboratório e Observatório da Mídia Esportiva. Publicado no Portal de Periódicos UFSC. As ideias expressadas neste artigo são de responsabilidade de seus autores, não representando, necessariamente, a opinião dos editores ou da universidade.

\section{EDITORES}

Mauricio Roberto da Silva, Giovani De Lorenzi Pires, Rogério Santos Pereira.

\section{HISTÓRICO}

Recebido em: 11 de Maio de 2019.

Aprovado em: 26 de Agosto de 2019. 